\title{
Bemerkungen.
}

Nov. 26. Comet schwach, verwaschen; etwa o.'5 Durchmesser, doch gut an hellen Fäden zu beobachten. - Dec. 2. Die Nähe des Vergleichsterns störte die Auffassung des Cometen, daher wurden die Beobachtungen bald abgebrochen; $\Delta \delta$ sehr unsicher. - Dec. 3. Comet recht gut zu sehen, etwa I' Durchmesser. - Dec. 4. Die unmittelbare Nähe eines Sterns $\mathrm{I}^{\mathrm{m}}$ störte die Beobachtung. - Dec. 8. Comet trotz Mondscheins und leichter Cirrostratus gut zu sehen, verwaschen, über 2' Durchmesser, rund, central verdichtet.

Die Helligkeit war durchschnittlich die eines Sterns $\mathrm{II}^{\mathrm{m}}-\mathrm{r} 2^{\mathrm{tn}}$, am 26 . Nov. nahe an $\mathrm{II}^{\mathrm{m}}$, am 4. Dec. geringer als $\mathrm{I}^{1} 1^{1 / 2}$, am 8. Dec. $\mathrm{I}^{\mathrm{m}}$. Vergr. 60 f., am 26. Nov. $80 \mathrm{f}$.

Jena II, I896 Dec. 9.

W. Winkler.

\section{Ephemeride für den periodischen Cometen Brooks (1889 V) 1896 VI.}

Fortsetzung zu A. N. 3389.

$12^{\text {h }}$ M. Z. Berlin.

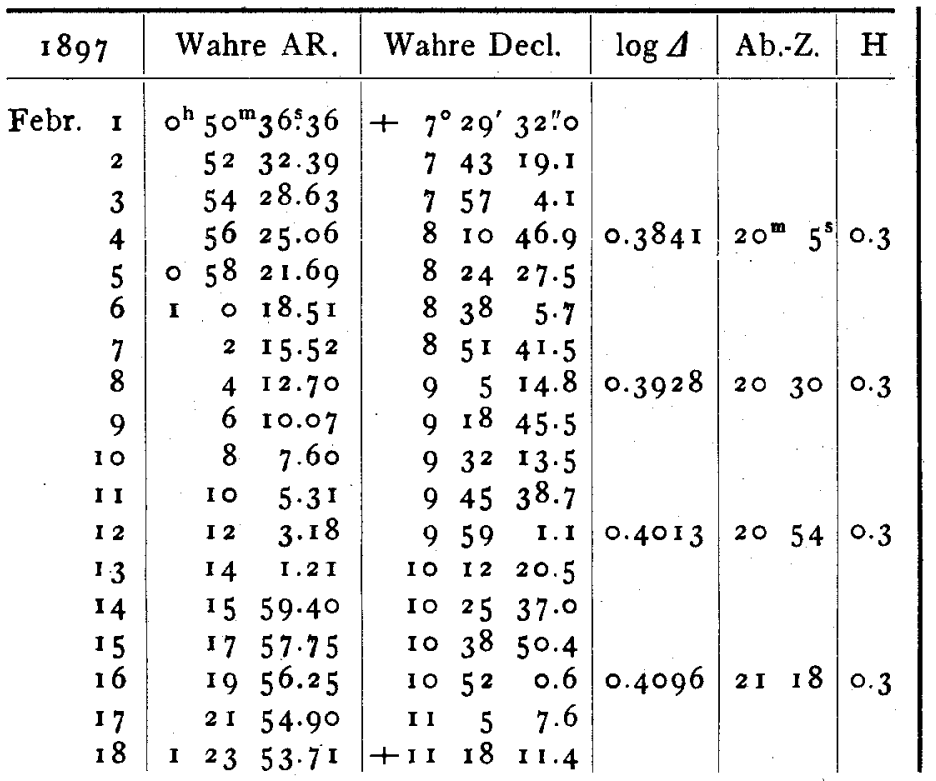

Berlin 1897 Jan. 27.

\begin{tabular}{|c|c|c|c|c|c|c|c|}
\hline \multicolumn{2}{|c|}{1897} & Wahre AR. & \multicolumn{2}{|c|}{ Wahre Decl. } & $\log A$ & Ab. $-Z$. & $\mathrm{H}$ \\
\hline Febr. & & $1^{h} 25^{m} 5^{2^{s}} \cdot 67$ & $+11^{\circ} 31^{\prime}$ & I I":8 & & & \\
\hline & 20 & 2751.77 & I I 44 & 8.8 & 0.4177 & $21^{\mathrm{m}} 42^{\mathrm{s}}$ & 0.3 \\
\hline & $2 \mathbf{I}$ & 2951.02 & I 57 & 2.4 & & & \\
\hline & 22 & 3 I 50.43 & I 29 & $5^{2 \cdot 5}$ & & & \\
\hline & 23 & $33 \quad 49.97$ & 1222 & 39.0 & & & \\
\hline & 24 & $35 \quad 49.67$ & 1235 & 21.9 & 0.4255 & 22 & 0.3 \\
\hline & 25 & 3749.52 & 1248 & I. I & & & \\
\hline & 26 & 3949.51 & I 30 & $3^{6.6}$ & & & \\
\hline & 27 & $41 \quad 49.65$ & I 3 I 3 & 8.3 & & & \\
\hline & 28 & $43 \quad 49.92$ & I 325 & 36.2 & $0.433^{2}$ & 2230 & 0.3 \\
\hline März & $\mathbf{I}$ & $45 \quad 50.34$ & 1338 & 0.0 & & & \\
\hline & 2 & $47 \quad 50.89$ & I 350 & 19.9 & & & \\
\hline & 3 & 4951.58 & I 42 & $35 \cdot 7$ & & & \\
\hline & 4 & $\begin{array}{ll}51 & 52.39\end{array}$ & 1414 & $47 \cdot 4$ & 0.4407 & 2253 & 0.2 \\
\hline & 5 & $53 \quad 53.33$ & 1426 & 54.9 & & & \\
\hline & 6 & $55 \quad 54.40$ & $143^{8}$ & 58.1 & & & \\
\hline & 7 & $57 \quad 55.59$ & 1450 & $5^{6.9}$ & & & \\
\hline & 8 & I $59 \quad 56.90$ & +152 & 51.4 & 0.4480 & $23 \quad 16$ & 0.2 \\
\hline
\end{tabular}

F. Bauschinger.

Neue Elemente des Cometen 1896 ...(Perrine Dec. 8).

Die Elemente beruhen auf drei Normalörtern 1896 Dec. I 0.5 (7 Beob.), I 896 Dec. 28.5 (6 Beob) und 1897 Jan. 5.5 (I 4 Beob.), zu denen ich die mir bekannt gewordenen Beobachtungen zusammengezogen habe. Dieselben stellen eine Nizzaer Beobachtung vom I2. Januar bis auf $\Delta \alpha=+0.18, \Delta \delta=-0.3$ dar.

Die Elemente lauten:

$$
\begin{aligned}
& T=1896 \text { Nov. } 24.65674 \text { M. Z. B. } \\
& \omega=163^{\circ} 53^{\prime} 30^{\prime \prime} \circ \\
& \left.\delta=24^{6} 34 \quad 35.9\right\} \quad \text { I } 897.0 \\
& i=134025.9 \\
& \varphi=424717.0 \\
& \log e=9.832054 \\
& \log q=0.045405 \\
& \mu=550.901 \\
& a=3.4617 \\
& U=6.44 I \text { Jahre }
\end{aligned}
$$

Heliocentrische Aequatorealcoordinaten.

$$
\begin{aligned}
& x=\left[9.9^{8} 9534^{6}\right] r \cdot \sin \left(v+139^{\circ} 5 \mathrm{I}^{\prime} 4 \mathrm{I}^{\prime \prime} 40\right) \\
& y=[9.9794489] r \cdot \sin (z+455049.2 \mathrm{I}) \\
& z=[9.5689189] r \cdot \sin (v+8342 \text { 3.13) }
\end{aligned}
$$

Der Comet nimmt in den heliocentrischen Längen $216^{\circ}$ und $247^{\circ}$ dieselben Radienvectoren an, die dort der Jupitersbahn zugehören, das erste Mal $30^{1 / 2}$ Grad vor dem aufsteigenden Knoten, das zweite Mal nur 1/2 Grad nach dem Durchgang durch den aufsteigenden Knoten. Hier kann die grösste Annäherung an Jupiter bis auf 0.053 stattfinden. Seit 1852 ist das zwar nicht eingetreten - da der Comet dann in kleinerer nördlicher heliocentrischer Breite dicht unter Jupiter durchgeht, so wäre eine starke Aenderung der Neigung, also eine viel schlechtere Uebereinstimmung der Elemente mit denen des Cometen Biela das Resultat -, wohl aber haben im Jahre 1888 grosse Jupiterstörungen stattgefunden. Als damals am 27. April der Comet durch 
diesen Ort grösster Bahnnähe ging, befand sich Jupiter nur $8 \mathrm{Grad}$ in Länge von demselben entfernt und besonders nach dem Knotendurchgang des Cometen war dieser noch längere Zeit den Jupiterstörungen ausgesetzt. Eine Identität der Cometen Perrine und Biela ist bei der um 60 Grad verschiedenen Richtung der grossen Axen ausgeschlossen. Eine Beziehung zwischen beiden, die durch das fast völlige Zusammenfallen der Babnebenen so sebr wabrscheinlich gemacht wird, kann man wohl nur so herstellen, dass man eine weitere Theilung des Cometen Biela nach $185_{2}$ oder eine frühere Theilung eines Hauptcometen in die Cometen Biela und Perrine annimmt. Nur müsste die Kraft, die den Cometen in zwei Theile geschieden hat, nicht wie bei der Theilung des Cometen Biela 1845 in der Richtung der Bahnlinie, sondern mehr in der Richtung des Radiusvectors wirksam angenommen werden, so dass die eine Componente in schräger Richtung nach aussen, die andere in schräger Richtung nach innen von der alten Bahnlinie weglief unter Beibehaltung der Bahnebene. Im Falle der Theilung eines Urcometen in früherer Zeit hätte also dessen Bahn zwischen den Bahnen der Cometen Perrine und Biela gelegen und nur der Punkt, wo damals die. Theilung erfolgte, wäre als Durchschnittspunkt allen drei Bahnen gemein. Nimmt man aber eine weitere Theilung des Cometen Biela nach 1852 an, so musste der Comet Perrine nach aussen unter Vergrösserung der Periheldistanz und Verkleinerung der Perihellänge, der Rest des Cometen Biela aber unter Verkleinerung der Periheldistanz und Vergrösserung der Perihellänge nach innen entweichen, und in der Bielabahn wäre von dem einen der beiden Cometentheile von 1845 resp. $185^{2}$ nichts mehr geblieben. Letztere Hypothese wird sich prüfen lassen, indem man untersucht, ob zu einer Zeit zwischen $185^{2}$ und 1896 die Cometen Biela und Perrine gleichzeitig in einem

\begin{tabular}{|c|c|c|c|c|c|}
\hline 1897 & $\alpha$ app. & $\delta$ app. & $\log r$ & $\log A$ & $\mathrm{H}$ \\
\hline Febr. 20 & $5^{\mathrm{h}} 5^{6^{\mathrm{m}}} 54^{\mathrm{s}}$ & $+2^{\circ} 23 ! 2$ & 0.19768 & $9.9455^{8}$ & 0.046 \\
\hline 22 & $\begin{array}{lll}6 & \text { I } & \text { I } 8\end{array}$ & $2 \quad 35 \cdot 3$ & & & \\
\hline $\begin{array}{l}24 \\
26\end{array}$ & $\begin{array}{ll}5 & 39 \\
9 & 56\end{array}$ & $\begin{array}{ll}2 & 47.1 \\
2 & 58.6\end{array}$ & 0.20673 & 9.97214 & 0.039 \\
\hline 28 & I 4 I I & 39.8 & 0.21563 & 9.99787 & 0.033 \\
\hline März 2 & 1823 & 320.6 & & & \\
\hline 4 & $22 \quad 33$ & 331.0 & $0.2244^{6}$ & 0.02293 & 0.028 \\
\hline 6 & $264 I$ & 341.0 & & & \\
\hline 8 & 3046 & 350.6 & 0.23313 & 0.04723 & 0.024 \\
\hline IO & $\begin{array}{lll}6 & 34 & 49\end{array}$ & +359.8 & & & \\
\hline
\end{tabular}

Durchschnittspunkt ihrer Bahnen haben stehen können. Die jetzige Bahn des Cometen Perrine und die Bielabahn von 1852 schneiden sich für die Argumente der Breite I6Iㄴ. und $14 \% 6$, doch kann nur der erstere Durchschnittspunkt in Betracht kommen, in welchem der Sinn der Bewegung in beiden Bahnen der gleiche ist, während in dem andern sich Comet Biela von der Sonne entfernt, Comet Perrine sich ihr aber nähert. Dem ersten Durchschnittspunkt entspricht für den Cometen Biela die wahre Anomalie 297\%1, für den Cometen Perrine $357^{\circ} 2$. Es mag bemerkt werden, dass der Comet Biela zur Zeit seiner Theilung I 845 eine nur um wenige Grad kleinere Anomalie als die hier geforderte besass. Doch haben weitere Untersuchungen in dieser Frage erst die Berechnung der Jupiterstörungen des Cometen Perrine I 888 und eine genauere Bestimmung der Elemente überhaupt abzuwarten. Ich beabsichtige die Bearbeitung des Cometen Perrine definitiv zu übernehmen und dann auch seinen Beziehungen zum Biela'schen Cometen nachzugehen. Dringend zu wünschen ist dafür eine möglichst lange Ausdehnung der jetzigen Beobachtungen. Die in A. N. 3396 mitgetheilte Ephemeride erfordert nach obigen Elementen die Correctionen:

\begin{tabular}{lcc}
\multicolumn{1}{c}{897} & $\Delta \alpha$ & $\Delta \delta$ \\
Jan. 31 & $+7^{\mathrm{s}}$ & +1.1 \\
Febr. 4 & +10 & +1.3 \\
8 & +12 & +1.7 \\
12 & +14 & +1.9 \\
16 & +17 & +2.0 \\
20 & +22 & +1.8
\end{tabular}

Im Folgenden gebe ich noch eine Fortsetzung der Ephemeride für I $^{\text {h }}$ M. Z. B.

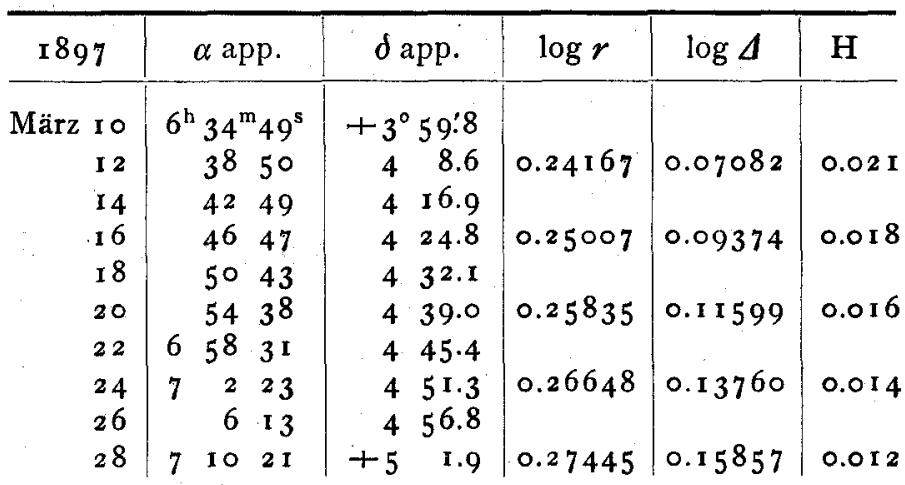

Mit obiger nach der Formel i : $r^{2} \Delta^{2}$ berechneten Helligkeit muisste der Comet, der zur Zeit der Entdeckung $8^{\mathrm{m}}$ war, am 28. März die dreizehnte Grösse besitzen, also noch zu beobachten sein, wenn nicht die Helligkeit, wie gewöhnlich, rascher abnimmt, als es diese Formel anzeigt.

Heidelberg, Königstuhl, I 897 Jan. 26.

F. Ristenpart.

\section{Ueber den Planeten Mars.}

I have just received a telegram from the Lowell Observatory now near the City of Mexico, with this information: „Rift in Martian north polar cap since January 7 , longitude $40^{\circ} . *$

Boston I 897 Jan, 10.

Fohn Ritchie jr. 